\title{
Iterative-Interpolated DFT for Synchrophasor Estimation: A Single Algorithm for P- and $\mathrm{M}^{-}$ Class Compliant PMUs
}

Article in IEEE Transactions on Instrumentation and Measurement · October 2017

DOI: 10.1109/TIM.2017.2779378

CITATIONS

0

3 authors:

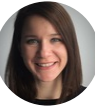

Asja Derviškadić

École Polytechnique Fédérale de Lausanne

6 PUBLICATIONS 3 CITATIONS

SEE PROFILE

\section{Mario Paolone}

École Polytechnique Fédérale de Lausanne

312 PUBLICATIONS 3,662 CITATIONS

SEE PROFILE
READS

53

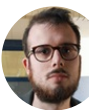

Paolo Romano

École Polytechnique Fédérale de Lausanne 23 PUBLICATIONS 206 CITATIONS

SEE PROFILE

Some of the authors of this publication are also working on these related projects:

Project

Project
Adaptive control of active distribution network integrating distributed resources View project

Development of Enhanced Phasor Measurements Units (PMUs) for Active Distribution Networks View project 


\title{
Iterative-Interpolated DFT for Synchrophasor Estimation: a Single Algorithm for P and M-class compliant PMUs
}

\author{
Asja Derviškadić, Member, IEEE, Paolo Romano, Member, IEEE, Mario Paolone, Senior Member, IEEE
}

\begin{abstract}
We present a single synchrophasor estimation (SE) algorithm that is simultaneously compliant with both $P$ and M Phasor Measurement Unit (PMU) performance classes. The method, called iterative-Interpolated DFT (i-IpDFT), iteratively estimates and compensates the effects of the spectral interference produced by both a generic interfering tone, harmonic or interharmonic, and the negative image of the fundamental tone. We define the 3-points i-IpDFT technique for cosine and Hanning window functions and we propose a procedure to select the iIpDFT parameters. We assess the performance of the i-IpDFT with respect to all the operating conditions defined in the IEEE Std. C37.118 for $P$ and M-class PMUs. We demonstrate that the proposed SE method is simultaneously compliant with all the accuracy requirements of both PMU performance classes.
\end{abstract}

Index Terms-Discrete Fourier transform (DFT), IEEE Std. C37.118, Interpolated Discrete Fourier transform (IpDFT), Phasor Measurement Unit (PMU), Synchrophasor Estimation.

\section{INTRODUCTION}

$\mathbf{T}$ HE IEEE Std. C37.118 [1] and its latest amendment [2] have defined two performance classes and related measurement requirements, to which Phasor Measurement Units (PMUs) must comply with: P-class is meant for applications requiring fast response time, such as power system protection; M-class is meant for high accuracy measurements requiring resiliency against interfering spectral components ${ }^{1}$. One of the main differences between $\mathrm{P}$ and $\mathrm{M}$-class requirements is represented by the Out-Of-Band Interference (OOBI) test. The latter, has been specifically designed for the M-class, to test the PMU capability to reject superposed interharmonics close to the 50 or $60 \mathrm{~Hz}$ main tone. These are defined as signals characterized by an amplitude that is $10 \%$ of the main tone and a frequency $f_{i}$ in the bands $\left[10, f_{n}-F_{r} / 2\right]$ and $\left[f_{n}+F_{r} / 2\right.$, $2 f_{n}$ ], being $f_{n}$ the nominal power system frequency, $F_{r}$ the PMU reporting rate and $f_{i} / f_{n} \notin \mathbb{N}[3]$.

Recently, the idea of a single PMU capable of satisfying both the $\mathrm{P}$ and M-class PMU requirements at the same time has become increasingly popular [4], [5]. The advantages are evident: from a cost perspective, an electrical utility interested to use PMUs to simultaneously supply monitoring

The Authors are with the École Polytechnique Fédérale de Lausanne EPFL, CH-1015, Lausanne, Switzerland.

${ }^{1}$ The amendment [2] introduces significant changes with respect to [1]. Therefore, the design of PMUs has evolved over time. Specifically, regarding frequency and Rate-Of-Change-Of-Frequency (ROCOF), [2] relaxes most of the requirements contained in [1] and suspends the ROCOF limit for the Out-Of-Band Interference (OOBI) test. Moreover, [2] changes and relaxes the requirements for measurement reporting latency and response time and simplifies the testing conditions for the frequency ramp test. and protection functionalities of situational awareness systems does not need to equip every measurement point with two different devices. From a measurement reliability perspective, the performance of fault management systems using PMU data to tune or back-up existing protection schemes, are not degraded by the presence of interharmonic tones [6]. In this respect, [4] proposes a hybrid $\mathrm{P}+\mathrm{M}$-class $\mathrm{PMU}$ design based on tunable trigger thresholds to switch between two different frequency-tracking filters. The technique self-switches from an $\mathrm{M}$-class algorithm to a P-class one in case a transient event is detected. On the contrary, [5] proposes a two-channel design that is conceived to comply with $\mathrm{P}$ and M-class at once. The process implements in parallel two different Taylor Fourier Transform algorithms, not designed to meet $\mathrm{P}$ and $\mathrm{M}$-class respectively: the first one produces accurate measurements of steady state signals, while the second one is better suited to follow fast signal changes. In this case, the selection of the most appropriate output is implemented by a detector that identifies the presence of power system transients.

In this paper, we propose a single algorithm designed to simultaneously fulfill the performance requirements of $\mathrm{P}$ and M-class PMUs. That is to say that, for each operating condition defined in [1], the method is compliant with the most demanding specification of the joint $\mathrm{P}$ and $\mathrm{M}$-class requirements. The technique is hereafter called iterative Interpolated Discrete Fourier Transform (i-IpDFT) and demonstrates how it is possible to develop a DFT-based SE algorithm capable of rejecting interharmonics, also when adopting relatively short window lengths ( 3 periods of a signal at $f_{n}$ ). The method represents an evolution of those proposed in [7] and [8], which are conceived for $\mathrm{P}$ and M-class PMUs, respectively. In this paper, we extend our investigation to meet simultaneously the compliance with $\mathrm{P}$ and $\mathrm{M}$-class requirements. To do so, we employ the 3-points IpDFT for cosine and Hanning window functions.

The i-IpDFT method iteratively finds and compensates the effects of the spectral interference produced by both an interfering tone and the negative image of the fundamental tone. Several algorithms that compensate the spectral interference generated by the image component of the main tone have been presented in the literature, the majority relying on the IpDFT [7]-[12]. Sine-fitting techniques [13], as well as compressive sensing [14] and lookup tables [15] have been investigated too. In [16] an IpDFT-based method that compensates the spectral interference generated by both the image component of the main tone and harmonic tones is 
presented. To the best of the Authors knowledge, [16] is the only contribution that takes into account tones other than the fundamental one, however such investigation disregards interharmonic tones like those defined in the OOBI test.

The paper is structured as follows: Section II reviews the theoretical background about the 3-points IpDFT for $\cos ^{\alpha}$ windows and presents the effects of spectral leakage on the IpDFT performance. Section III formulates the i-IpDFT SE algorithm, with a specific focus on the selection of the algorithm parameters. Then Section IV assesses the algorithm performance in an offline simulation environment with respect to the testing conditions defined in [1]. A dedicated computational complexity is also presented in order to support the prospective integration of the algorithm into an embedded hardware. Finally, Section V concludes the paper with final remarks.

\section{INTERPOLATED DFT FOR $\cos ^{\alpha}$ WINDOWS}

The IpDFT is a technique that enables us to extract the parameters of a sinusoidal waveform by processing the highest DFT bins of the related DFT spectrum. In particular, the IpDFT enables us to mitigate the effects generated by incoherent sampling by [17], [18]:

- Applying suitably shaped window functions to reduce the spectral leakage effects;

- Interpolating the highest DFT bins of the spectrum to minimize the spectral sampling effects.

In the following of this Section we present the IpDFT technique and define the nomenclature. More specifically, in Section II-A we introduce the family of $\cos ^{\alpha}$ window functions with a particular focus on the cosine and the Hanning (or Hann) window. These two window types have been selected as they represent a good trade-off between the main-lobe width and the side-lobe levels. In Section II-B we derive the IpDFT solution for these window functions. Finally, in Section II-C we discuss the effects of spectral leakage on the IpDFT performance.

\section{A. $\cos ^{\alpha}$ Window Functions}

The IpDFT solution can be analytically derived only for $\cos ^{\alpha}$ windows (also known as $\sin ^{\alpha}$ windows) ${ }^{2}$, that are defined as follows [19]:

$$
w_{\alpha}(n)=\sin ^{\alpha}\left(\frac{\pi}{N} n\right), \quad n \in[0, N-1], \quad \alpha \in \mathbb{N}
$$

being $N$ the number of samples. In particular, Fig. 1 shows three different $\cos ^{\alpha}$ window functions obtained for $\alpha=$ $\{0,1,2\}$. As it can be noted, the window spectrum depends upon $\alpha$ : as $\alpha$ decreases, the main lobe shrinks but the side lobes increase, whereas as $\alpha$ increases, the side lobes decrease but the main lobe becomes wider.

Generally, IpDFT algorithms were formulated for the so called Rife-Vincent class I windows, i.e., $\cos ^{\alpha}$ windows characterized by a null or even value for $\alpha$. The most elementary

\footnotetext{
2 The IpDFT can be formulated for window functions not belonging to the $\cos ^{\alpha}$ family. However, this problem does not have a closed form analytical solution. In that case, the solution may use approximations of the numerically computed window spectrum.
}
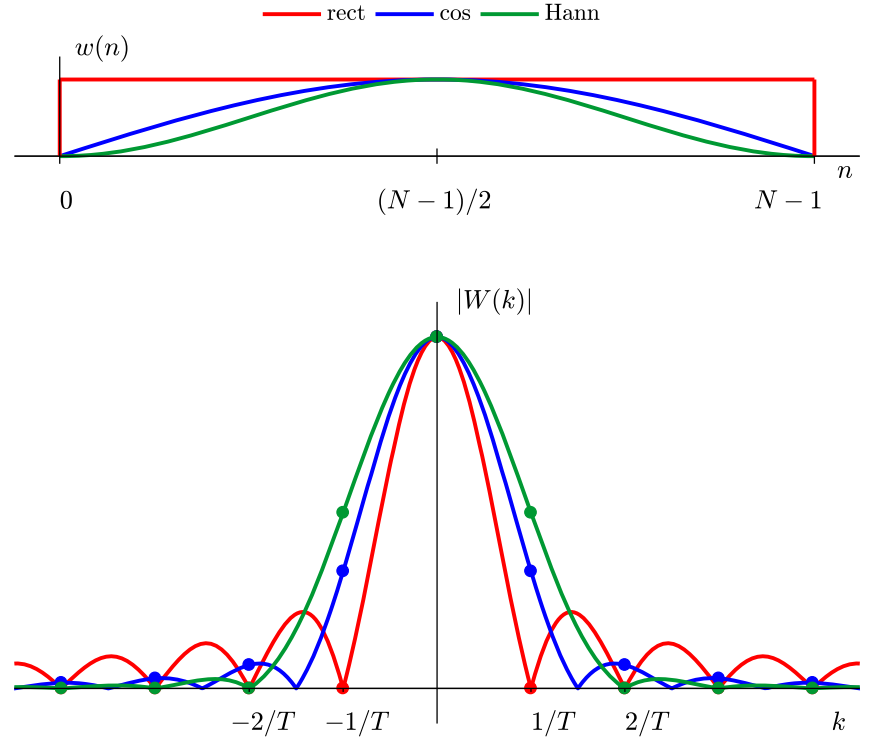

Fig. 1. Time (top) and frequency domain (bottom) representations of three different $\cos ^{\alpha}$ windows: the rectangular $(\alpha=0)$, cosine $(\alpha=1)$ and Hanning $(\alpha=2)$.

one is the rectangular $(\alpha=0)$, whose DFT is known as the Dirichlet kernel:

$$
D_{N}(k)=e^{-j \pi k(N-1) / N} \frac{\sin (\pi k)}{\sin (\pi k / N)}, k \in[0, N-1]
$$

This function is characterized by the narrowest main lobe among the $\cos ^{\alpha}$ windows but at the same time it exhibits the highest side lobes.

In order to reduce the effects of spectral leakage generated by the side-lobe levels, IpDFT algorithms typically adopt bellshaped windows, obtained by increasing the value of $\alpha$ in (1). The most common is the Hanning window $(\alpha=2)$ defined as:

$$
w_{H}(n)=0.5 \cdot(1-\cos (2 \pi n / N)), \quad n \in[0, N-1]
$$

and characterized by the following DFT:

$W_{H}(k)=-0.25 \cdot D_{N}(k-1)+0.5 \cdot D_{N}(k)-0.25 \cdot D_{N}(k+1)$

that is known for the good trade-off between the main-lobe width and side-lobe levels [18].

More recent studies have derived the analytical solution of the IpDFT problem for $\cos ^{\alpha}$ windows in the case of an odd value for $\alpha$ [20]. In applications where the main lobe width plays a crucial role in identifying relatively nearby tones, like in SE, the so-called cosine window, defined for $\alpha=1$, is an extremely attractive solution as it represents a compromise between the rectangular and the Hanning window:

$$
w_{C}(n)=\sin \left(\frac{\pi}{N} n\right), n \in[0, N-1]
$$

Its DFT can be derived as follows:

$$
W_{C}(k)=-0.5 j D_{N}(k-0.5)+0.5 j D_{N}(k+0.5)
$$

However, for an odd value for $\alpha$, the window is a weighted sum of Dirichlet kernels modulated by non-integers of the 


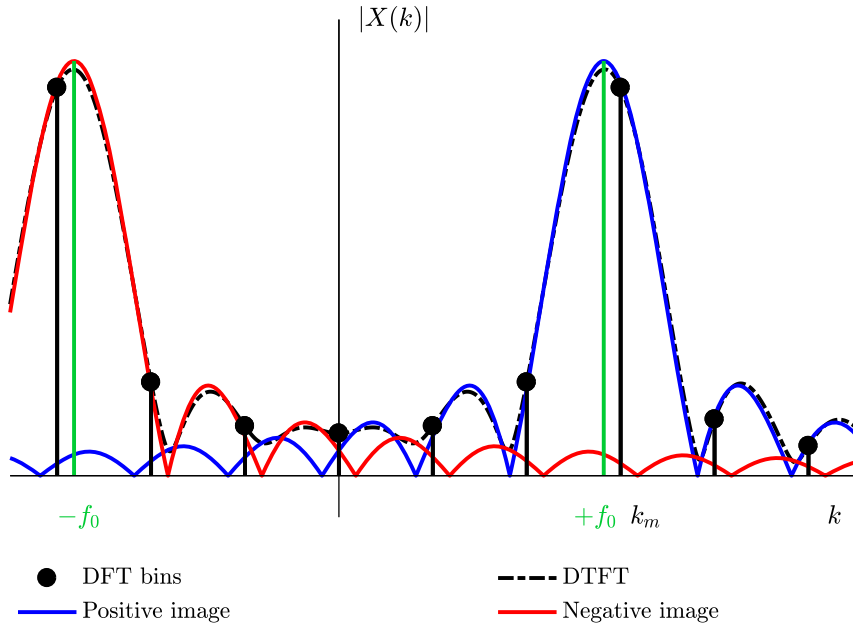

Fig. 2. DFT and DTFT of a steady state single-tone signal that is incoherently sampled and the related positive and negative images.

frequency resolution, therefore an intrinsic limitation arises: as it can be seen from Fig. 1, the $W_{C}(k)$ zero-crossing do not happen for integer values of $k$. As a consequence, also with coherent sampling, the cosine window generates spectral leakage.

\section{B. The Interpolated DFT (IpDFT)}

As known, the IpDFT is based on a static signal model that, in general, is assumed to contain a single unknown frequency component. In this respect, let us consider a finite sequence of $N$ equally spaced samples $x(n)$ obtained by sampling an input continuous signal $x(t)$ with a sampling rate $F_{s}=1 / T_{s}$, being $T_{s}$ the sampling time:

$$
x(n)=A_{0} \cos \left(2 \pi f_{0} n T_{s}+\varphi_{0}\right), \quad n \in[0, N-1]
$$

where $\left\{f_{0}, A_{0}, \varphi_{0}\right\}$ are the signal frequency, amplitude and initial phase respectively. The signal is assumed to be windowed with a discrete function of $N$ samples $w(n)$, being $T=N \cdot T_{s}$ the window length. The DFT $X(k)$ of the windowed input signal is:

$$
X(k) \triangleq \frac{1}{B} \sum_{n=0}^{N-1} w(n) x(n) W_{N}^{k n}, \quad k \in[0, N-1]
$$

where $B \triangleq \sum_{n=0}^{N-1} w(n)$ is the DFT normalization factor, and $W_{N} \triangleq e^{-j 2 \pi / N}$ is the so called twiddle factor. The DFT returns a sequence of $N$ samples (also called bins) of the theoretical Discrete Time Fourier Transform (DTFT) that are uniformly spaced by the DFT frequency resolution $\Delta_{f}=1 / T$. More specifically, based on the convolution theorem, the DFT of the windowed signal $x(n)$ exhibits a pair of scaled, shifted and rotated versions of the DFT of the window function (see Fig. 2): the so-called positive image (in blue), is shifted up to the tone frequency $f_{0}$, the so-called negative image (in red), is shifted down to $-f_{0}$ :

$$
X(k)=X_{0}^{+}(k)+X_{0}^{-}(k)
$$

where:

$$
\left\{\begin{array}{l}
X_{0}^{+}(k)=A_{0} e^{+j \varphi_{0}} W\left(k-f_{0} / \Delta_{f}\right) \\
X_{0}^{-}(k)=A_{0} e^{-j \varphi_{0}} W\left(k+f_{0} / \Delta_{f}\right)
\end{array}\right.
$$

being $W(k)$ the DFT of the adopted window function.

As shown in Fig. 2, in case of incoherent sampling $\left(f_{0} / \Delta_{f} \notin \mathbb{N}\right)$, the peak value of the continuous spectrum of the fundamental tone of a signal (highlighted in green) is located between two consecutive DFT bins and the signal frequency can be expressed as:

$$
f_{0}=\left(k_{m}+\delta\right) \Delta_{f}
$$

being $-0.5 \leq \delta<0.5$ a fractional correction term and $k_{m}$ the index of the highest bin. The IpDFT problem lies in finding the correction term $\delta$ (and, consequently, the fundamental tone's parameters $\left\{f_{0}, A_{0}, \varphi_{0}\right\}$ ) that better approximates the exact location of the main spectrum tone.

The IpDFT problem solution has been originally provided as a 2-point interpolation scheme [17], [18]. More recently, multipoint interpolation schemes have demonstrated to inherently reduce the long-term spectral leakage effects, leading to more accurate interpolation results [10], [11]. In this respect, for the Hanning window, the fractional term $\delta$ can be computed by interpolating the 3 highest DFT bins as [10]:

$$
\delta=2 \varepsilon \cdot \frac{\left|X\left(k_{m}+\varepsilon\right)\right|-\left|X\left(k_{m}-\varepsilon\right)\right|}{\left|X\left(k_{m}-\varepsilon\right)\right|+2\left|X\left(k_{m}\right)\right|+\left|X\left(k_{m}+\varepsilon\right)\right|}
$$

where $\varepsilon= \pm 1$ if $\left|X\left(k_{m}+1\right)\right| \gtrless\left|X\left(k_{m}-1\right)\right|$. The fundamental tone's amplitude and phase can then be computed as:

$$
\begin{aligned}
& A_{0}=\left|X\left(k_{m}\right)\right|\left|\frac{\pi \delta}{\sin (\pi \delta)}\right|\left|\delta^{2}-1\right| \\
& \varphi_{0}=\angle X\left(k_{m}\right)-\pi \delta
\end{aligned}
$$

Similarly, for the cosine window, $\delta$ can be obtained by interpolating the 3 highest DFT bins as [20]:

$$
\delta=1.5 \varepsilon \cdot \frac{\left|X\left(k_{m}+\varepsilon\right)\right|-\left|X\left(k_{m}-\varepsilon\right)\right|}{\left|X\left(k_{m}-\varepsilon\right)\right|+2\left|X\left(k_{m}\right)\right|+\left|X\left(k_{m}+\varepsilon\right)\right|}
$$

The fundamental tone's amplitude and phase can then be computed as (computational details are given in Appendix):

$$
\begin{aligned}
& A_{0}=4 \cdot \frac{\left|\delta^{2}-0.25\right|}{|\cos (\pi \delta)|} \cdot\left|X\left(k_{m}\right)\right| \\
& \varphi_{0}=\angle X\left(k_{m}\right)-\pi \delta
\end{aligned}
$$

\section{Spectral Leakage Effects on the IpDFT}

The main assumptions behind the formulation of the IpDFT technique are the following [17]:

1) The input signal is characterized by time-invariant parameters;

2) The input signal is sampled with a sampling rate sufficiently higher than the highest signal's spectral component;

3) The DFT bins used to perform the interpolation are only generated by the positive image of the tone under analysis.

In order to satisfy the first two assumptions when applying the IpDFT to SE, sampling rates in the order of few $\mathrm{kHz}$ and 
window lengths containing few periods of a signal at the rated power system frequency must be adopted respectively [21]. This choice causes the energy of the DFT spectrum to be concentrated in the lower frequency range and the positive and negative image of the main tone of the spectrum to be relatively close. In such conditions, in case of incoherent sampling, the tails of the negative image of the spectrum main tone (red curve in Fig. 2) leak into the positive frequency range and bias the DFT bins used to perform the interpolation (i.e., assumption 3 is not respected). This effect, also known as spectral interference has demonstrated to considerably corrupt the IpDFT estimations when applied to SE [12]. To cope with these conditions, in [7] a technique that mitigates the effect of the spectral leakage produced by the negative image of the spectrum is presented. The method, called enhanced-IpDFT (e-IpDFT) is described by Algorithm 1, where the function DFT refers to (8), IPDFT refers to (11)-(14) and wf to (10a) or $(10 b)$.

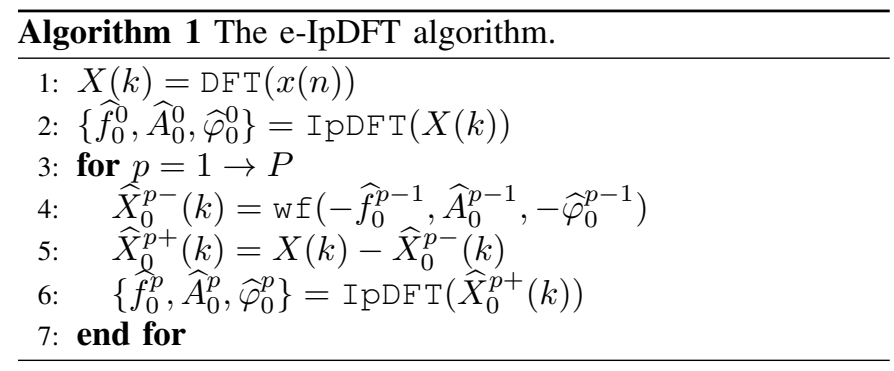

It starts with the computation of the DFT spectrum $X(k)$ (line 1), that can be performed with any DFT computation method $^{3}$. Then, a preliminary estimation of the main tone parameters is obtained by applying the IpDFT to $X(k)$ (line 2). These values are used to estimate the main tone's negative image $\widehat{X}_{0}^{-}(k)$ (line 4$)$, as in (10b), that is then subtracted from $X(k)$, to return an estimation of the main tone's positive image (line 5). The IpDFT is applied to the resulting spectrum, where the spectral interference produced by the negative image is considerably reduced (line 6). The compensation of the spectral interference produced by the negative image of the fundamental tone, can be improved by iterating the procedure a predefined number of times $P$ (see Section III-B). We summarize the method proposed in [7] by defining a single function e-IPDFT that accounts for lines 2-7 of Algorithm 1:

$$
\left.\left\{\widehat{f}_{0}, \widehat{A}_{0}, \widehat{\varphi}_{0}\right\}\right|_{P}=\mathrm{e}-\operatorname{IpDFT}[X(k)]
$$

The method presented in [7] does not account for the spectral interference produced by tones other than the fundamental one. As a consequence, it produces incorrect results in presence of interfering tones that are relatively close to the main one, such those defined in the OOBI test.

\section{The Proposed ITERATIVE IPDFT (I-IPDFT)}

This Section aims at presenting the i-IpDFT, that represents an enhancement of the method proposed in [7] as it takes

\footnotetext{
${ }^{3}$ Recursive DFT computation methods that update the DFT spectrum on a sample basis are normally more efficient when calculating a small amount of DFT bins, such in the case of the e-IpDFT algorithm (e.g., [22]).
}

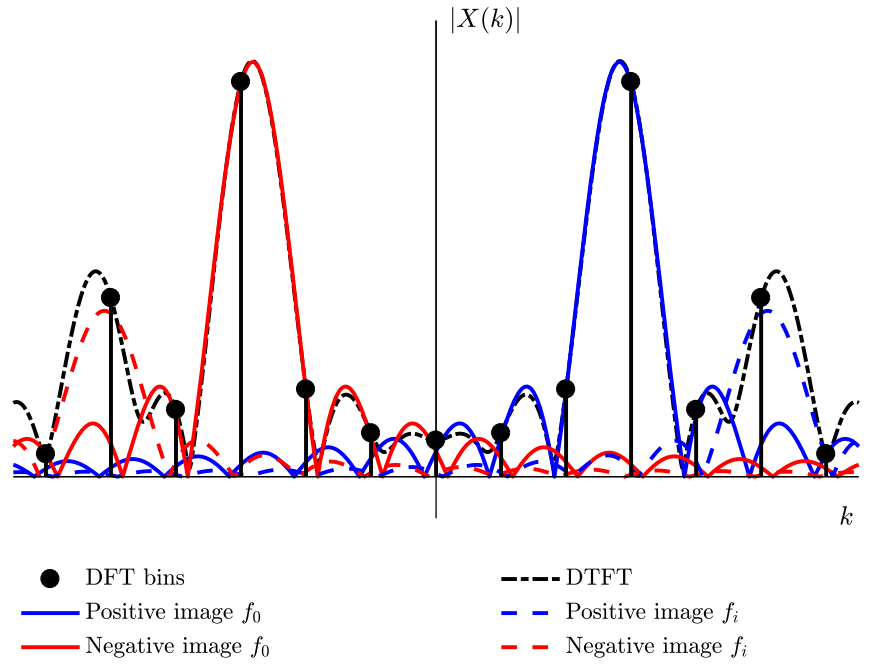

Fig. 3. DFT and DTFT of a steady state signal composed by a main tone and an interharmonic one and the related positive and negative images.

into account the effects of the spectral interference generated by both the negative image of the main tone and a generic interfering one. More specifically, in Section III-A we define the SE algorithm and in Section III-B and III-C we propose a procedure to tune the algorithm parameters.

\section{A. The i-IpDFT Algorithm Formulation}

Let us consider a steady-state discrete signal composed of two tones, a fundamental and an interfering tone (not necessary harmonic, i.e., $f_{i} / f_{0} \in \mathbb{R}$ ), both unknown:

$$
x(n)=A_{0} \cos \left(2 \pi f_{0} n T_{s}+\varphi_{0}\right)+A_{i} \cos \left(2 \pi f_{i} n T_{s}+\varphi_{i}\right)
$$

As shown in Fig. 3, its DFT spectrum can be modelled as the sum of the positive (in blue) and negative (in red) images of the fundamental (solid line) and interfering (dashed line) tones:

$$
\begin{aligned}
X(k) & =X_{0}(k)+X_{i}(k) \\
& =X_{0}^{+}(k)+X_{0}^{-}(k)+X_{i}^{+}(k)+X_{i}^{-}(k)
\end{aligned}
$$

The proposed i-IpDFT algorithm iteratively estimates and compensates the effects of spectral leakage generated by an interfering tone and by the negative image of the main tone, such that the IpDFT is applied to a DFT spectrum that is only composed by the positive image of the main tone $X_{0}^{+}(k)$. The pseudo-code of the proposed i-IpDFT is reported in Algorithm 2, whereas Fig. 4 illustrates the spectra that are processed during successive steps of the method.

The first steps (lines 1-2) of the i-IpDFT algorithm exactly correspond to the e-IpDFT technique in Algorithm 1. Although in presence of an interfering tone, the estimated main tone parameters might be largely biased, they can be used to approximate the positive and the negative image of the fundamental tone (line 3, Fig. 4b). The latter are then subtracted from the original DFT bins to produce a spectrum $X(k)-\widehat{X}_{0}(k)$ that accounts for any spurious contribution perturbing the fundamental tone of the signal (line 4, Fig. 4c). 


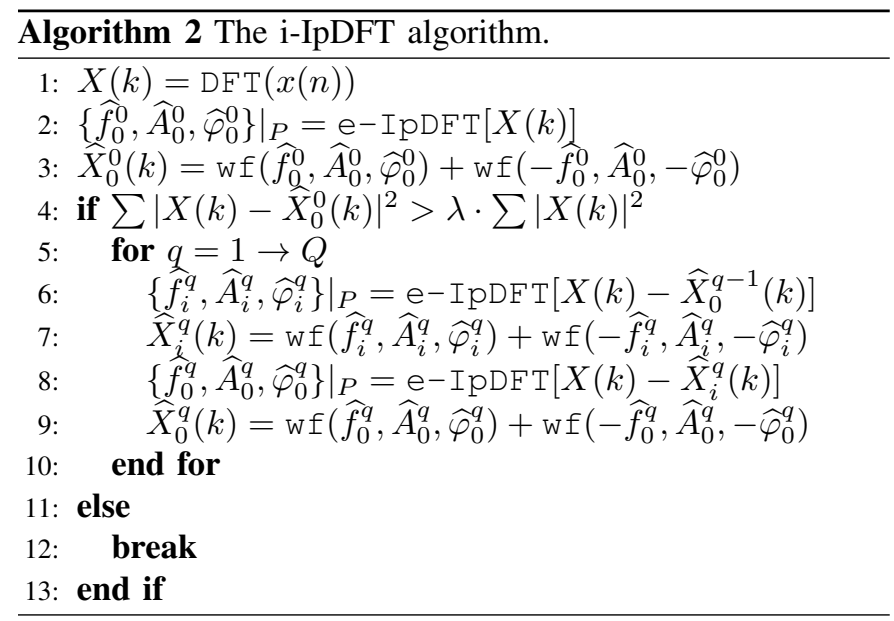

In case an interfering tone is detected, the procedure that estimates and compensates its spectral interference must be activated. Otherwise the method stops and returns the parameters $\left\{\widehat{f}_{0}^{0}, \widehat{A}_{0}^{0}, \widehat{\varphi}_{0}^{0}\right\}$ estimated at line 2 . If an interfering tone is present, the spectral energy of $X(k)-\widehat{X}_{0}^{0}(k)$ weighted by the spectral energy of $X(k)$, exceeds a certain threshold $\lambda$ (see Section III-C for further details):

$$
E_{n}=\frac{E\left[X(k)-\widehat{X}_{0}^{0}(k)\right]}{E[X(k)]}=\frac{\sum_{k=0}^{K}\left|X(k)-\widehat{X}_{0}^{0}(k)\right|^{2}}{\sum_{k=0}^{K}|X(k)|^{2}}>\lambda
$$

where $K$ is the total number of computed DFT bins. In such a case, the e-IpDFT is applied to $X(k)-\widehat{X}_{0}^{q-1}(k)$, to estimate the parameters $\left\{\widehat{f}_{i}^{q}, \widehat{A}_{i}^{q}, \widehat{\varphi}_{i}^{q}\right\}$ of the detected interharmonic tone (line 6). The latter, are used to evaluate both the positive and negative image of the interharmonic tone (line 7, Fig. 4d), that are then subtracted from the original DFT bins, obtaining the spectrum $X(k)-\widehat{X}_{i}^{q}(k)$ that does not contain the interfering tone (line 8, Fig. 4e). Finally, the e-IpDFT is applied to such spectrum leading to an enhanced estimation of the main tone parameters $\left\{\widehat{f}_{0}^{q}, \widehat{A}_{0}^{q}, \widehat{\varphi}_{0}^{q}\right\}$ (line 8, Fig. 4f).

The whole procedure can be iterated a predefined number of times $Q$, leading to more and more accurate estimates as $Q$ increases (see Section III-B). The presented i-IpDFT algorithm can be formulated for any window function, number of IpDFT interpolation points, window length and sampling frequency. Even though it has been formulated for a single interfering component, it can be easily extended to consider more than one interfering component. In this respect, it is worth mentioning that the amount of DFT bins to be calculated at line 1 depends on the highest frequency component that has to be compensated.

\section{B. On the Tuning of the Number of Iterations $P$ and $Q$}

The performance of the i-IpDFT algorithm are mainly influenced by two parameters, and in what follows a procedure to select them is presented:

- $P$ : the number of iterations of the compensation of the spectral interference generated by the negative image of the tone under analysis (see Algorithm 1);

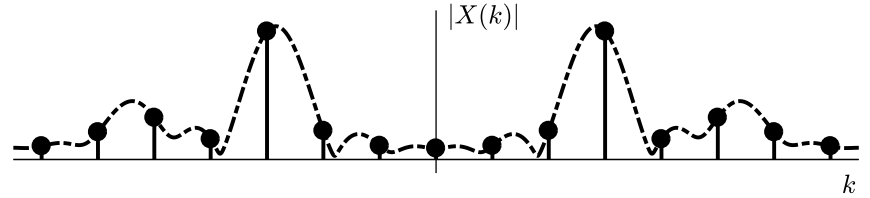

(a)

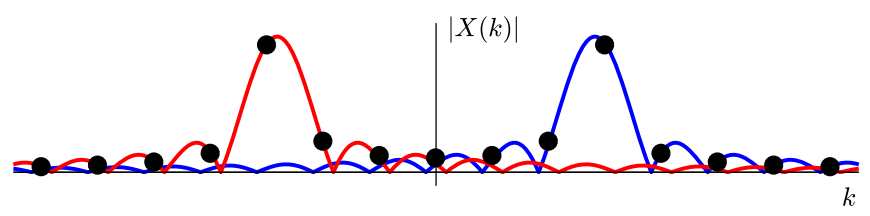

(b)

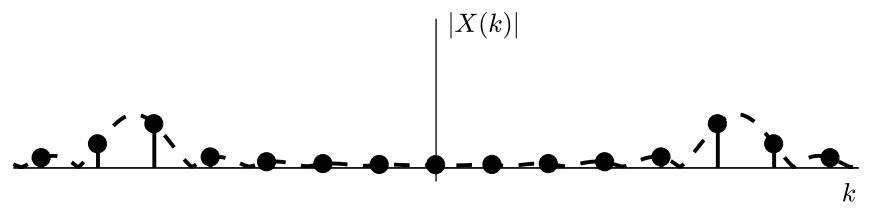

(c)

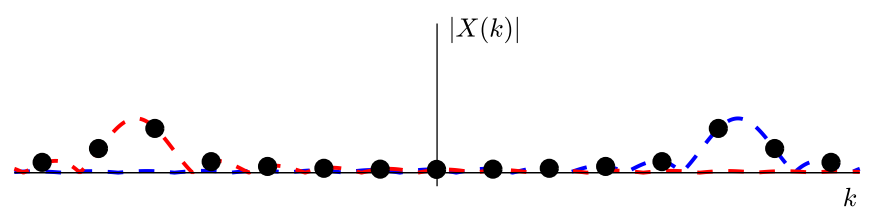

(d)

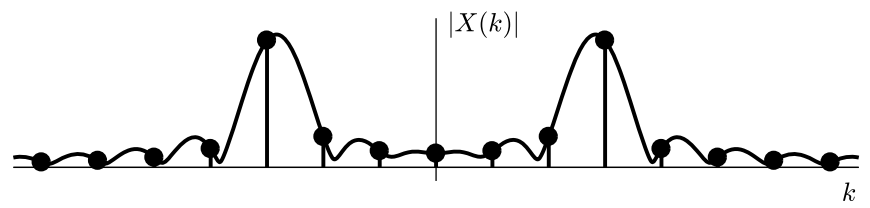

(e)

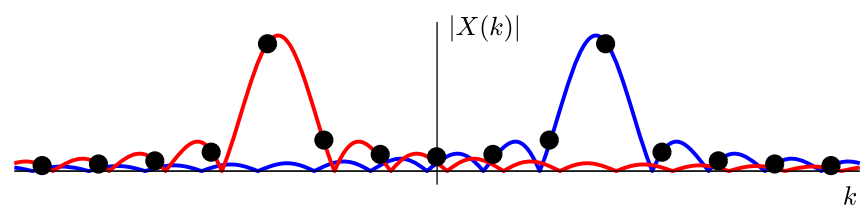

(f)

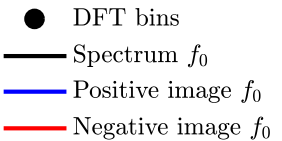

---D DTFT

- - Spectrum $f_{i}$

- - Positive image $f_{i}$

- - Negative image $f_{i}$

Fig. 4. Spectra processed at different steps of Algorithm 2: $X(k)$ in line 1 (a); $\widehat{X}_{0}(k)^{0}$ in line $3(\mathrm{~b}) ; X(k)-\widehat{X}_{0}^{q-1}(k)$ in line 4 and $6(\mathrm{c}) ; \widehat{X}_{i}^{q}(k)$ in line $7(\mathrm{~d}) ; X(k)-\widehat{X}_{i}^{q}(k)$ in line $8(\mathrm{e}) ; \widehat{X}_{0}^{q}(k)$ in line $9(\mathrm{f})$.

- $Q$ : the number of iterations of the overall procedure (see Algorithm 2).

The effects of $P$ can be evaluated when applying the e-IPDFT technique to a single-tone signal that is incoherently sampled. In this respect, Fig. 5 shows the accuracy in estimating the IpDFT correction term $\delta$ as a function of $P$, when analyzing a signal at $47.5 \mathrm{~Hz}$ with a sampling rate of $50 \mathrm{kHz}$ and a window length of $60 \mathrm{~ms}$. In order to emulate more realistic conditions, the signal is characterized by an $80 \mathrm{~dB}$ Signal-to-Noise Ratio (SNR) [23]. As it can be noted, in this specific case, the effect of the compensation 


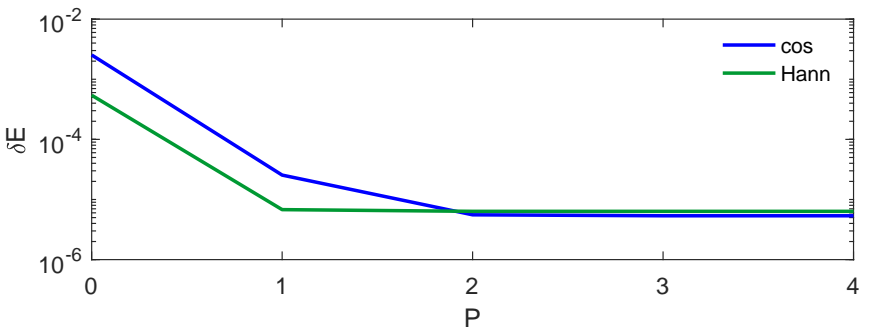

Fig. 5. Error in estimating the IpDFT correction term $\delta$ with the e-IpDFT technique, as a function of $P$ : signal at $f_{0}=47.5 \mathrm{~Hz} . F_{s}=50 \mathrm{kHz}, T=$ $60 \mathrm{~ms}, \mathrm{SNR}=80 \mathrm{~dB}$.

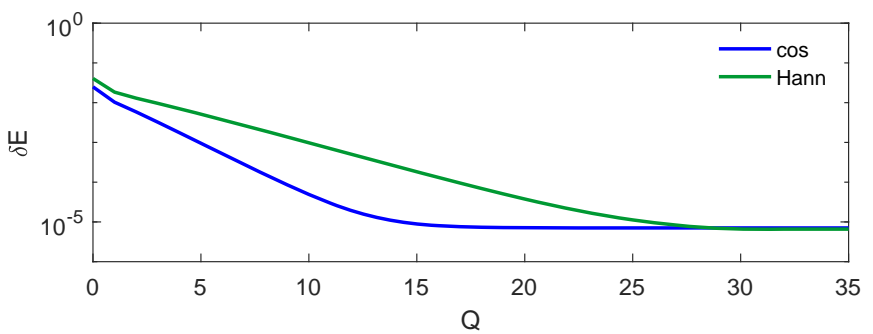

Fig. 6. Error in estimating the IpDFT correction term $\delta$ with the i-IpDFT technique, as a function of $Q$ : signal at $f_{0}=47.5 \mathrm{~Hz}$ corrupted by an interfering tone at $f_{i}=20 \mathrm{~Hz} . F_{s}=50 \mathrm{kHz}, T=60 \mathrm{~ms}, \mathrm{SNR}=80 \mathrm{~dB}$.

becomes negligible after $P=2$ iterations both for the Hanning and the cosine windows. In general this is not always the case and the number of iterations gets higher as the relative distance between the positive and negative images gets smaller. Nevertheless, due to its good trade-off between the overall algorithm performance and computational complexity, in the following of the paper the results will be presented for $P=2$.

The effects of the overall number of iterations $Q$ are evaluated when applying the i-IpDFT algorithm to a signal corrupted by an interharmonic tone. In particular, the case of a signal characterized by a main tone at $47.5 \mathrm{~Hz}$ and an interharmonic tone at $20 \mathrm{~Hz}$ is presented (similar results hold for all combinations of $f_{0}$ and $f_{i}$ in the OOBI range). Again, an $80 \mathrm{~dB}$ SNR is considered [23]. Fig. 6 shows the error in estimating the correction term $\delta$ with the i-IpDFT technique $\left(F_{s}=50 \mathrm{kHz}, T=60 \mathrm{~ms}\right)$ as a function of $Q$. For the cosine window the effects of the compensation become negligible after $Q=16$ iterations, whereas for the Hanning window after $Q=28$ iterations. This result is explained by the fact that the narrower main lobe of the cosine window allows to detect (and therefore compensate) the interharmonic tone with a smaller number of iterations. As a consequence, in the following of this paper, the i-IpDFT results are presented for $Q=16$ and $Q=28$ for the cosine and Hanning window respectively.

\section{On the tuning of the threshold $\lambda$}

The threshold $\lambda$ must be set so that the iterative compensation (i.e., lines 5-10 of Algorithm 2) is activated only in presence of an interharmonic tone. Furthermore, the proposed technique can turn out to be extremely useful to compensate the spectral leakage generated by an harmonic tone when using the cosine window, which has demonstrated to generate spectral leakage also with coherent sampling.
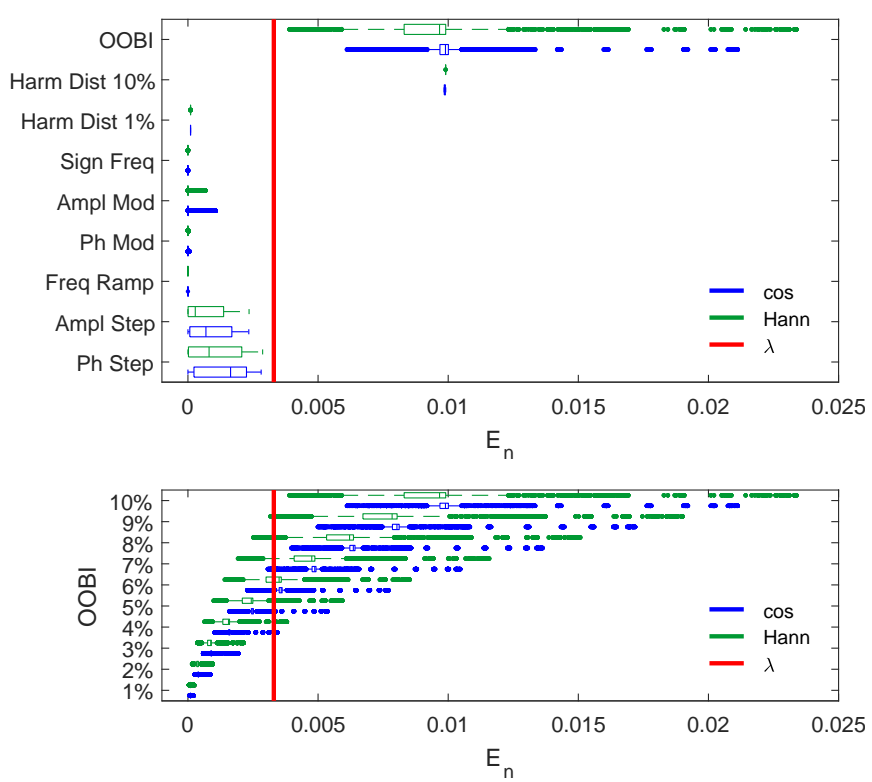

Fig. 7. Boxplot representation of $E_{n}$ in all operating conditions indicated in [1] (top) and in the OOBI test when changing $A_{i}$ in the range $[1,10] \% A_{0}$ (bottom). $F_{s}=50 \mathrm{kHz}, f_{n}=50 \mathrm{~Hz}, T=60 \mathrm{~ms}, \mathrm{SNR}=80 \mathrm{~dB}$.

In general, the normalized spectral energy $E_{n}$ can contain contributions generated by an interfering tone (e.g., harmonic or interharmonic) as well as any spurious component generated by a dynamic event that is wrongly captured by the DFT (e.g., steps in amplitude and in phase or amplitude and phase modulations). In this respect, Fig. 7 shows the variability of $E_{n}$ by means of a boxplot representation for the various testing conditions dictated by [1]. Fig. 7 shows that, when the main tone is corrupted by a $10 \%$ harmonic or interharmonic component, the values of $E_{n}$ are relatively higher than all the other testing conditions. Therefore, in the following of this paper, the value of $\lambda$ is set to $3.3 \cdot 10^{-3}$, in order to activate the algorithm only in presence of interfering components higher than $10 \%$ of the fundamental tone.

Even if not required by [1], we carry out dedicated simulations to assess the influence of lower interharmonic amplitudes on the i-IpDFT performance. Specifically, we vary $A_{i}$ within the interval $[1,10] \% A_{0}$. As it can be seen from Fig. 7, the threshold $\lambda=3.3 \cdot 10^{-3}$ enables the correct identification of interharmonic tones characterized by $A_{i}$ above $8 \%$ for the cosine window and 9\% for the Hanning one. Consequently, a correct behavior of the algorithm takes place and the errors are below the limits dictated by [1]. Below such amplitudes, given that the iterative compensation in not activated, the i-IpDFT cannot identify and correct the interharmonic tone. Therefore, the errors exceed the limits dictated by [1]. Even though a lower value of $\lambda$ would enable the detection of interfering tones characterized by smaller amplitudes, it would not enable the distinction between an interfering tone (to be eliminated from the signal spectrum) and a dynamic behavior (not to be eliminated). 
TABLE I

I-IPDFT PARAMETERS.

\begin{tabular}{lcc}
\hline \multicolumn{1}{c}{ Parameter } & Variable & Value \\
\hline Nominal system frequency & $f_{n}$ & $50 \mathrm{~Hz}$ \\
Window length & $T$ & $60 \mathrm{~ms}\left(3 / f_{n}\right)$ \\
Sampling rate & $F_{s}$ & $50 \mathrm{kHz}$ \\
PMU reporting rate & $F_{r}$ & $50 \mathrm{fps}$ \\
DFT bins & $K$ & 11 \\
Iterations comp. of the image & $P$ & 2 \\
Iterations overall procedure & $Q$ & $16(\cos ), 28$ (Hann) \\
IpDFT interpolation points & - & 3 \\
\hline
\end{tabular}

\section{Performance Assessment}

We carry out the numerical validation of the i-IpDFT algorithm in a simulation environment, by making reference to the static and dynamic performance requirements dictated by [1], [2] and following the testing procedures described in the IEEE Guide C37.242 [24]. In order to limit the number of tests, the nominal frequency and the reporting rate have been fixed to $50 \mathrm{~Hz}$ and 50 frames-per-second (fps) respectively. For each test, the results are presented by means of three graphs showing the maximum Total Vector Error (TVE), Frequency Error (FE) and ROCOF Error (RFE) as a function of the independent variable of the specific test, together with the maximum limit allowed by [1] for both $\mathrm{P}$ and M-class PMU (Fig. 8 - 15). Moreover, two tables summarize the maximum obtained TVE, FE and RFE and the maximum limit allowed by [1] in all tests (Tables II and III). Although [1] does not provide any guidelines regarding the noise, additive white Gaussian noise with zero mean and variance corresponding to an SNR in the range $[60,80] \mathrm{dB}$ is added to the various reference signals, in order to simulate more realistic conditions [23]. For the sake of clarity, since the trend of errors is similar for any value of SNR, the graphs refer to an SNR of $80 \mathrm{~dB}$, whereas the tables include the maximum obtained errors for both 60 and $80 \mathrm{~dB}$.

As resumed in Table I, the $\mathrm{i}$-IpDFT algorithm results are shown for both the Hanning (green lines) and cosine windows (blue lines), using a sampling rate of $50 \mathrm{kHz}$ and a window containing 3 periods of a signal at the nominal power system frequency. This is the shortest observation interval that enables us to distinguish between the fundamental and the interfering tone, and to fulfill jointly the $\mathrm{P}$ and M-class requirements ${ }^{4}$. The time-stamp of the synchrophasor is referred to the center of the applied window. The synchrophasors are estimated at overlapping observation windows, that are shifted by the given PMU reporting rate. The first 10 DFT bins of the spectrum are computed, in order to be able to compensate the effects of any interfering component up to the $3^{\text {rd }}$ harmonic. The 3-points IpDFT is used to estimate the fractional correction term $\delta$. It is worth pointing out that the parameters resumed in Table I are kept constant during all tests, and that such

\footnotetext{
${ }^{4}$ The existing literature on DFT-based SE techniques has discussed algorithms characterized by shorter observation intervals. However, those estimators are performing well only in quasi-stationary conditions and when the signal frequency is neither far from the nominal one nor corrupted by interfering tones (e.g., [25]).
}
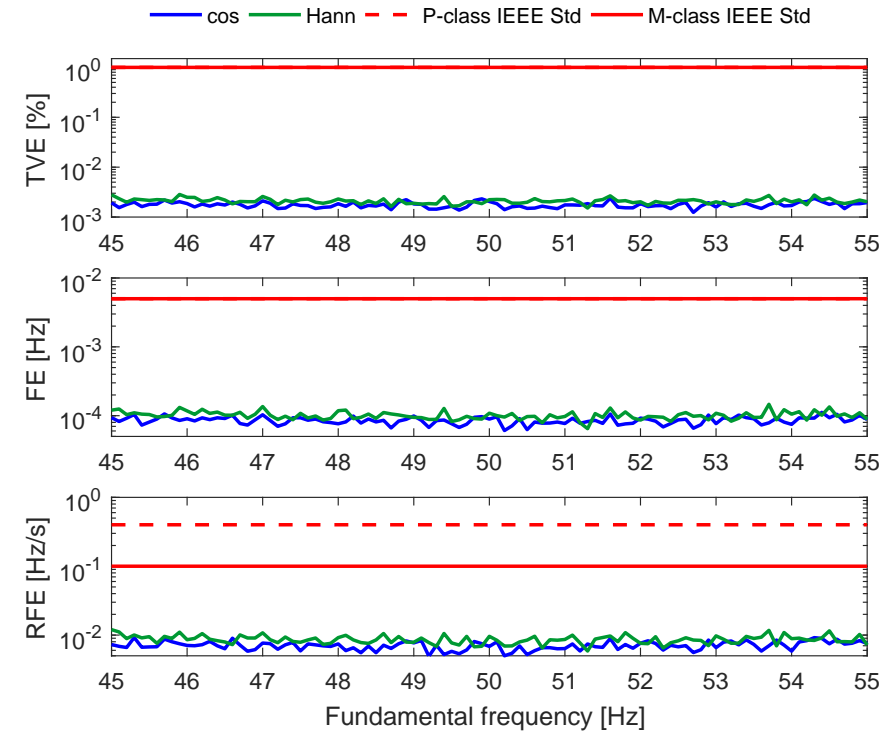

Fig. 8. Signal frequency test [1].

configuration enables us to simultaneously meet the P and Mclass compliance in all operating conditions defined in [1].

Finally, the Rate-Of-Change-Of-Frequency (ROCOF) is computed by means of a classical backward first-order approximation of a first-order derivative:

$$
\operatorname{ROCOF}(n)=\left|\widehat{f}_{0}(n)-\widehat{f}_{0}(n-1)\right| \cdot F_{r}
$$

where $\widehat{f}_{0}(n)$ and $\widehat{f}_{0}(n-1)$ represent the fundamental frequency estimations at two successive reporting times.

\section{A. Static Conditions}

Regarding the steady state conditions (see Fig. 8, 9, 10 and Table II), three cases are analyzed according to [1]:

- Signal frequency: single-tone signals in the frequency range $45 \leq f_{0} \leq 55 \mathrm{~Hz}$ (Fig. 8);

- Harmonic distortion: signals distorted by a single $10 \%$ harmonic in the frequency range $100 \leq f_{h} \leq 2500 \mathrm{~Hz}$ (Fig. 9)

- OOBI: signals characterized by a fundamental frequency $f_{0}$ of $47.5,50$ and $52.5 \mathrm{~Hz}$, distorted by single $10 \%$ interharmonics in the frequency range $10 \leq f_{i} \leq 25 \mathrm{~Hz}$ and $75 \leq f_{i} \leq 100 \mathrm{~Hz}$ (Fig. 10).

During the signal frequency test (see Fig. 8), the errors are not influenced by the fundamental tone frequency. As it can be noted from Table II, when the wideband noise level is $60 \mathrm{~dB}$, in case of using the Hanning window, the RFE is $0.126 \mathrm{~Hz} / \mathrm{s}$, being $0.1 \mathrm{~Hz} / \mathrm{s}$ the maximum limit allowed by [1] Nevertheless, in the same conditions, for the cosine window the RFE is below such limit. Regarding the harmonic distortion test (see Fig. 9), in case the Hanning window is adopted, the errors are invariant with respect to the harmonic order, due to the fact that both the fundamental and the harmonic tone are coherently sampled. On the contrary, for the cosine window the two tones are not coherently sampled, therefore the effects of spectral leakage are significant, worsening the 
TABLE II

COMPLIANCE WITH RESPECT TO STATIC SIGNALS: MAXIMUM TVE, FE AND RFE FOR THE I-IPDFT AND MAXIMUM LIMIT ALLOWED BY [1].

\begin{tabular}{|c|c|c|c|c|c|c|c|c|c|c|c|c|c|c|c|c|c|c|}
\hline & \multicolumn{6}{|c|}{ TVE [\%] } & \multicolumn{6}{|c|}{$\mathrm{FE}[\mathrm{mHz}]$} & \multicolumn{6}{|c|}{ RFE [Hz/s] } \\
\hline & \multicolumn{2}{|c|}{ IEEE Std } & \multicolumn{4}{|c|}{ i-IpDFT } & \multicolumn{2}{|c|}{ IEEE Std } & \multicolumn{4}{|c|}{ i-IpDFT } & \multicolumn{2}{|c|}{ IEEE Std } & \multicolumn{4}{|c|}{ i-IpDFT } \\
\hline & \multirow[t]{2}{*}{$\mathrm{P}$} & \multirow[t]{2}{*}{ M } & \multicolumn{2}{|c|}{$\begin{array}{c}\cos \\
\text { SNR }[\mathrm{dB}]\end{array}$} & \multicolumn{2}{|c|}{$\begin{array}{c}\text { Hann } \\
\text { SNR }[\mathrm{dB}]\end{array}$} & \multirow[t]{2}{*}{$\mathrm{P}$} & \multirow[t]{2}{*}{$\mathrm{M}$} & \multicolumn{2}{|c|}{$\begin{array}{c}\cos \\
\text { SNR }[\mathrm{dB}]\end{array}$} & \multicolumn{2}{|c|}{$\begin{array}{c}\text { Hann } \\
\text { SNR }[\mathrm{dB}]\end{array}$} & \multirow[t]{2}{*}{$\mathrm{P}$} & \multirow[t]{2}{*}{ M } & \multicolumn{2}{|c|}{$\begin{array}{c}\cos \\
\text { SNR }[\mathrm{dB}]\end{array}$} & \multicolumn{2}{|c|}{$\begin{array}{c}\text { Hann } \\
\text { SNR }[\mathrm{dB}]\end{array}$} \\
\hline & & & 60 & 80 & 60 & 80 & & & 60 & 80 & 60 & 80 & & & 60 & 80 & 60 & 80 \\
\hline Sign $F$ & 1 & 1 & 0.024 & 0.002 & 0.03 & 0.003 & 5 & 5 & 1.3 & 0.1 & 1.5 & 0.1 & 0.4 & 0.1 & 0.095 & 0.009 & 0.126 & 0.012 \\
\hline Harm Dist $1 \%$ & 1 & 1 & 0.108 & 0.094 & 0.028 & 0.003 & 5 & 25 & 5.4 & 4.7 & 1.3 & 0.1 & 0.4 & - & 0.086 & 0.009 & 0.112 & 0.011 \\
\hline Harm Dist $10 \%$ & 1 & 1 & 0.055 & 0.047 & 0.026 & 0.003 & 5 & 25 & 2 & 1.1 & 1.2 & 0.1 & 0.4 & - & 0.085 & 0.009 & 0.124 & 0.011 \\
\hline$f_{0}=47.5 \mathrm{H}$ & - & 1.3 & 0.056 & 0.022 & 0.108 & 0.08 & - & 10 & 2.7 & 1.1 & 5.6 & 4. & - & - & 0.217 & 0.101 & 0.513 & 0.369 \\
\hline OOBI $f_{0}=50 \mathrm{~Hz}$ & - & 1.3 & 0.026 & 0.003 & 0.033 & 0.004 & - & 10 & 1.3 & 0.1 & 1.7 & 0.2 & - & - & 0.104 & 0.009 & 0.153 & 0.013 \\
\hline$f_{0}=52.5 \mathrm{~Hz}$ & - & 1.3 & 0.043 & 0.004 & 0.044 & 0.011 & - & 10 & 2.1 & 0.2 & 2.2 & 0.6 & - & - & 0.143 & 0.022 & 0.150 & 0.032 \\
\hline
\end{tabular}

TABLE III

COMPLIANCE WITH RESPECT TO DYNAMIC SIGNALS: MAXIMUM TVE, FE AND RFE FOR THE I-IPDFT AND MAXIMUM LIMIT ALLOWED BY [1].

\begin{tabular}{|c|c|c|c|c|c|c|c|c|c|c|c|c|c|c|c|c|c|c|}
\hline & \multicolumn{6}{|c|}{ TVE [\%] } & \multicolumn{6}{|c|}{$\mathrm{FE}[\mathrm{mHz}]$} & \multicolumn{6}{|c|}{$\mathrm{RFE}[\mathrm{Hz} / \mathrm{s}]$} \\
\hline & \multicolumn{2}{|c|}{ IEEE Std } & \multicolumn{4}{|c|}{ i-IpDFT } & \multicolumn{2}{|c|}{ IEEE Std } & \multicolumn{4}{|c|}{ i-IpDFT } & \multicolumn{2}{|c|}{ IEEE Std } & \multicolumn{4}{|c|}{ i-IpDFT } \\
\hline & \multirow[t]{2}{*}{$\mathrm{P}$} & \multirow[t]{2}{*}{ M } & \multicolumn{2}{|c|}{$\begin{array}{c}\cos \\
\text { SNR }[\mathrm{dB}]\end{array}$} & \multicolumn{2}{|c|}{$\begin{array}{c}\text { Hann } \\
\text { SNR [dB] }\end{array}$} & \multirow[t]{2}{*}{$\mathrm{P}$} & \multirow[t]{2}{*}{ M } & \multicolumn{2}{|c|}{$\begin{array}{c}\cos \\
\text { SNR }[\mathrm{dB}]\end{array}$} & \multicolumn{2}{|c|}{$\begin{array}{c}\text { Hann } \\
\text { SNR [dB] }\end{array}$} & \multirow[t]{2}{*}{$P$} & \multirow[t]{2}{*}{ M } & \multicolumn{2}{|c|}{$\begin{array}{c}\cos \\
\text { SNR }[\mathrm{dB}]\end{array}$} & \multicolumn{2}{|c|}{$\begin{array}{c}\text { Hann } \\
\text { SNR [dB] }\end{array}$} \\
\hline & & & 60 & 80 & 60 & 80 & & & 60 & 80 & 60 & 80 & & & 60 & 80 & & 80 \\
\hline Ampl Mod & 3 & 3 & 0.846 & 0.847 & 0.604 & 0.604 & 60 & 300 & 2.2 & 1.6 & 1.6 & 0.4 & 2.3 & 14 & 0.106 & 0.051 & 0.123 & 0.016 \\
\hline Ph Mod & 3 & 3 & 0.805 & 0.806 & 0.547 & 0.547 & 60 & 300 & 21.9 & 22 & 17.9 & 17.4 & 2.3 & 14 & 0.725 & 0.683 & 0.568 & 0.540 \\
\hline \multirow[t]{5}{*}{ Freq Ramp } & 1 & 1 & 0.058 & 0.055 & 0.044 & 0.038 & 10 & 10 & 1 & 0.2 & 0.9 & 0.2 & 0.4 & 0.2 & 0.088 & 0.011 & 0.083 & 0.011 \\
\hline & \multicolumn{6}{|c|}{ TVE Response time [s] } & \multicolumn{6}{|c|}{ FE Response time [s] } & \multicolumn{6}{|c|}{ RFE Response time [s] } \\
\hline & \multicolumn{2}{|c|}{ IEEE Std } & \multicolumn{4}{|c|}{ i-IpDFT } & \multicolumn{2}{|c|}{ IEEE Std } & \multicolumn{4}{|c|}{ i-IpDFT } & \multicolumn{2}{|c|}{ IEEE Std } & & i-Ip & DFT & \\
\hline & $P$ & M & $\begin{array}{r}c \\
\text { SNR }\end{array}$ & os & $\begin{array}{r}\mathrm{H} \\
\text { SNR }\end{array}$ & $\begin{array}{l}\text { nn } \\
{[\mathrm{dB}]}\end{array}$ & $\mathrm{P}$ & $\mathrm{M}$ & $\begin{array}{r}\mathrm{c} \\
\text { SNR }\end{array}$ & {$[\mathrm{dB}]$} & $\begin{array}{r}\mathrm{Ha} \\
\text { SNR }\end{array}$ & $\begin{array}{l}\mathrm{nn} \\
{[\mathrm{dB}]}\end{array}$ & $P$ & $\mathrm{M}$ & $\begin{array}{r}c \\
\text { SNR }\end{array}$ & $\begin{array}{l}\text { os } \\
{[\mathrm{dB}]}\end{array}$ & $\begin{array}{l}\mathrm{Ha} \\
\text { SNR }\end{array}$ & $\begin{array}{l}\mathrm{nn} \\
{[\mathrm{dB}]}\end{array}$ \\
\hline & & & 60 & 80 & 60 & 80 & & & 60 & 80 & 60 & 80 & & & 60 & 80 & 60 & 80 \\
\hline Ampl Step & 0.04 & 0.14 & 0.034 & 0.034 & 0.028 & 0.028 & 0.09 & 0.28 & 0.048 & 0.048 & 0.044 & 0.044 & 0.12 & 0.28 & 0.056 & 0.056 & 0.054 & 0.054 \\
\hline Ph Step & 0.04 & 0.14 & 0.040 & 0.040 & 0.032 & 0.032 & 0.09 & 0.28 & 0.048 & 0.048 & 0.044 & 0.044 & 0.12 & 0.28 & 0.054 & 0.054 & 0.054 & 0.054 \\
\hline & & & Delay & time $[\mathrm{s}]$ & & & & & Iax Ov & ershoot & [\%] & & & & & & & \\
\hline & IEEE & Std & & i-Ip & DFT & & IEEI & Std & & i-Ip & DFT & & & & & & & \\
\hline & $\mathrm{P}$ & M & $\begin{array}{r}c \\
\text { SNR }\end{array}$ & os & $\begin{array}{r}\mathrm{H}_{2} \\
\text { SNR }\end{array}$ & $\begin{array}{l}\mathrm{nn} \\
{[\mathrm{dB}]}\end{array}$ & $\mathrm{P}$ & M & $\begin{array}{r}c c \\
\text { SNR }\end{array}$ & s & $\begin{array}{r}\mathrm{Ha} \\
\text { SNR }\end{array}$ & $\begin{array}{l}\mathrm{nn} \\
{[\mathrm{dB}]}\end{array}$ & & & & & & \\
\hline & & & 60 & 80 & 60 & 80 & & & 60 & 80 & 60 & 80 & & & & & & \\
\hline Ampl Step & 0.005 & 0.005 & 0.002 & 0.002 & 0.002 & 0.002 & 5 & 10 & 0 & 0 & 0 & 0 & & & & & & \\
\hline Ph Step & 0.005 & 0.005 & 0.002 & 0.002 & 0.002 & 0.002 & 5 & 10 & 0 & 0 & 0 & 0 & & & & & & \\
\hline
\end{tabular}
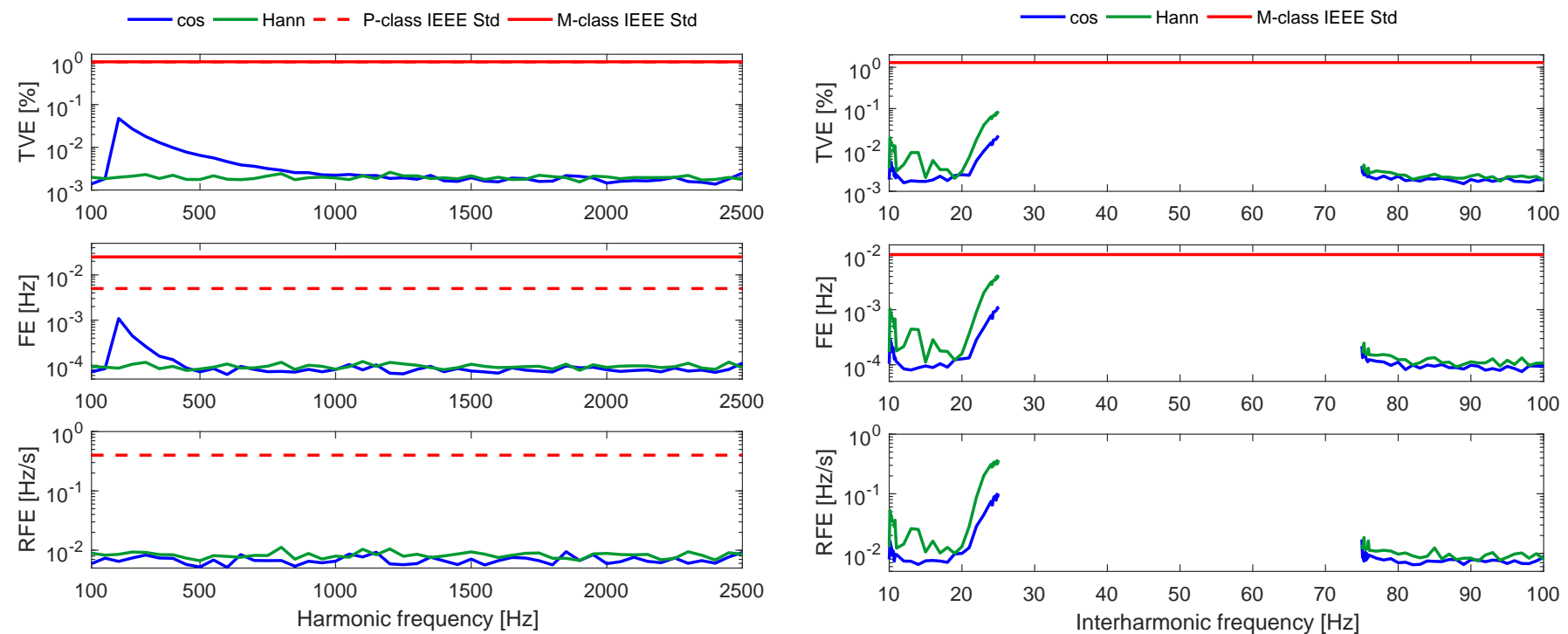

Fig. 9. Harmonic distortion test $\left(A_{h}=10 \% A_{0}\right)[1]$.

Fig. 10. OOBI test [1]. 
IpDFT results especially for lower harmonic orders. Indeed, as reported in Table II, in case $A_{h}=1 \% A_{0}$ the FE exceeds the $5 \mathrm{mHz}$ limit dictated by [1] when $\mathrm{SNR}=60 \mathrm{~dB}$, and is below such limit for higher SNRs. It is worth mentioning that, in case $A_{h}=10 \% A_{0}$, for the second and third harmonic the weighted energy $E_{n}$ exceeds the threshold $\lambda$, activating the procedure that compensates the detected harmonic tone, leading to smaller errors even for the cosine window.

As far as the OOBI test is concerned (see Fig. 10), the graphs show the maximum obtained errors among the 3 considered fundamental frequencies. The TVE and FE are within the limits required by [1] for every combination of interharmonic and fundamental frequencies, for both cosine and Hanning window. Even though [1] does not define any specific limit for the RFE, the obtained results show that we are capable of estimating the ROCOF with similar performance as in the harmonic distortion test. To be more precise, the most challenging condition is when the two tones have frequencies that are close together and their images are not detectable due to short-term spectral leakage. In case $f_{0}=47.5 \mathrm{~Hz}$, this occurs for $20 \leq f_{i} \leq 25 \mathrm{~Hz}$ whereas in case $f_{0}=52.5 \mathrm{~Hz}$, this occurs for $75 \leq f_{i} \leq 80 \mathrm{~Hz}$.

\section{B. Dynamic Conditions}

Regarding the dynamic conditions (see Fig. 11-15 and Table III), three cases are analyzed according to [1]:

- Measurement bandwidth: signals characterized by phase and amplitude modulations, being $0.1 \leq f_{m} \leq 5 \mathrm{~Hz}$ the modulating frequency (Fig. 11 and 12);

- Frequency ramp: signals characterized by positive and negative frequency ramps in the frequency range $48 \leq$ $f_{0} \leq 52 \mathrm{~Hz}$ and $45 \leq f_{0} \leq 55 \mathrm{~Hz}$ at a rate of $\pm 1 \mathrm{~Hz} / \mathrm{s}$ (Fig. 13);

- Amplitude and phase steps: signals characterized by positive and negative amplitude $( \pm 10 \%)$ and phase $( \pm \pi / 18)$ steps (Fig. 14 and 15).

During the measurement bandwidth test (see Fig. 11 for amplitude and Fig. 12 for phase modulation results), despite the errors increase with the modulating frequency, they are always well within the limits required by [1]. As for the Frequency ramp, the errors fully satisfy the requirements and are comparable with those obtained during the signal frequency test even within the exclusion intervals (Fig. 13 shows the positive frequency ramp for the $[45,55] \mathrm{Hz}$ range, similar results hold for the negative ramp and for the [48, 52] Hz range). Finally, regarding the amplitude and phase steps (see Fig. 14 and 15 for the positive step cases), the errors are represented as a function of the measured response time. Moreover, the estimated amplitude (A) and phase (ph) are represented as a function of the delay time. The results show that, during positive steps, the errors return below the limits within the allowed response times without any violation of the overshoot limit (similar results hold for the negative step cases).

\section{Computational Complexity}

In view of implementing the i-IpDFT algorithm into an FPGA-based (Field Programmable Gate Array) device, we

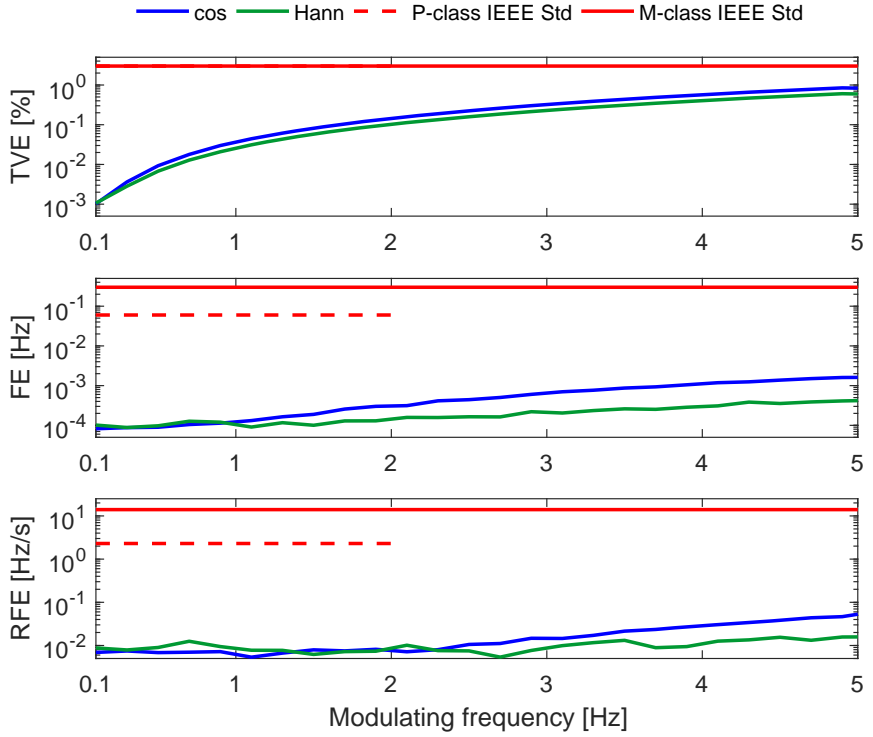

Fig. 11. Amplitude modulation test [1]. The range of the modulation frequency is $[0.1,2] \mathrm{Hz}$ for P-class, [0.1,5] for M-class [1].
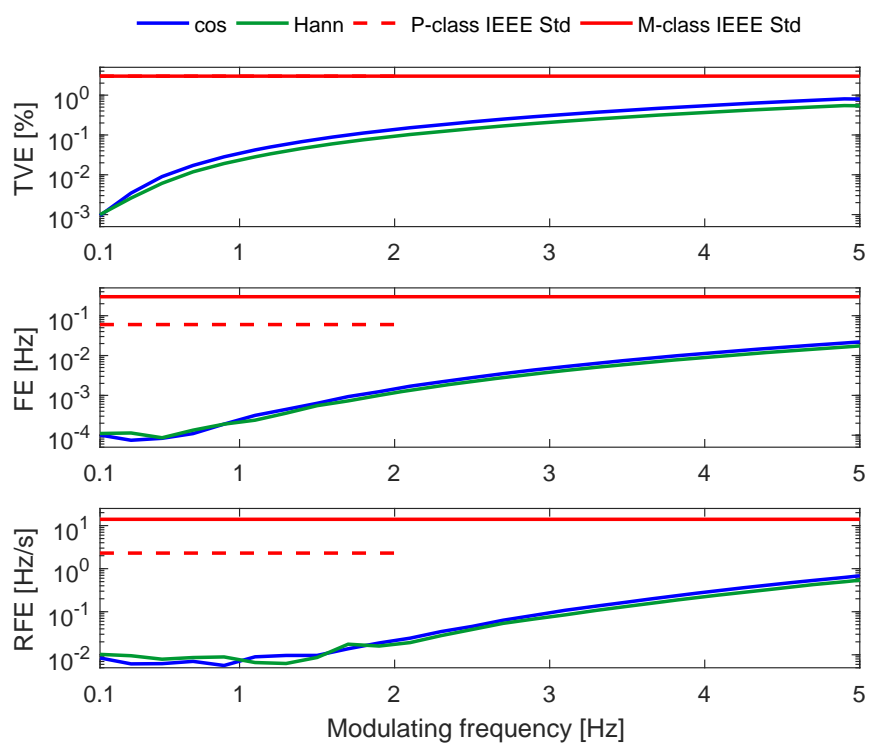

Fig. 12. Phase modulation test [1]. The range of the modulation frequency is $[0.1,2] \mathrm{Hz}$ for P-class, $[0.1,5]$ for M-class [1].

have assessed its computational complexity. More specifically, Table IV summarizes the number of arithmetic operations required to produce each step of the i-IpDFT, via the pseudocode shown in Algorithm 2.

Overall, the workload is higher than the one of the procedure presented in [7]. Nevertheless, the possibility to share FPGA resources when implementing iterative algorithms, such as the i-IpDFT, enables us to considerably reduce the overall FPGA occupation and potentially fit the proposed i-IpDFT in an embedded device. In this regard, [22] demonstrates that the e-IpDFT technique is taking less than 20 microseconds to estimate the synchrophasor of a single input channel in the targeted FPGA device. As summarized in Table IV, when implementing the i-IpDFT, for each estimated synchrophasor, the e-IpDFT is applied $Q$ times to both the fundamental 

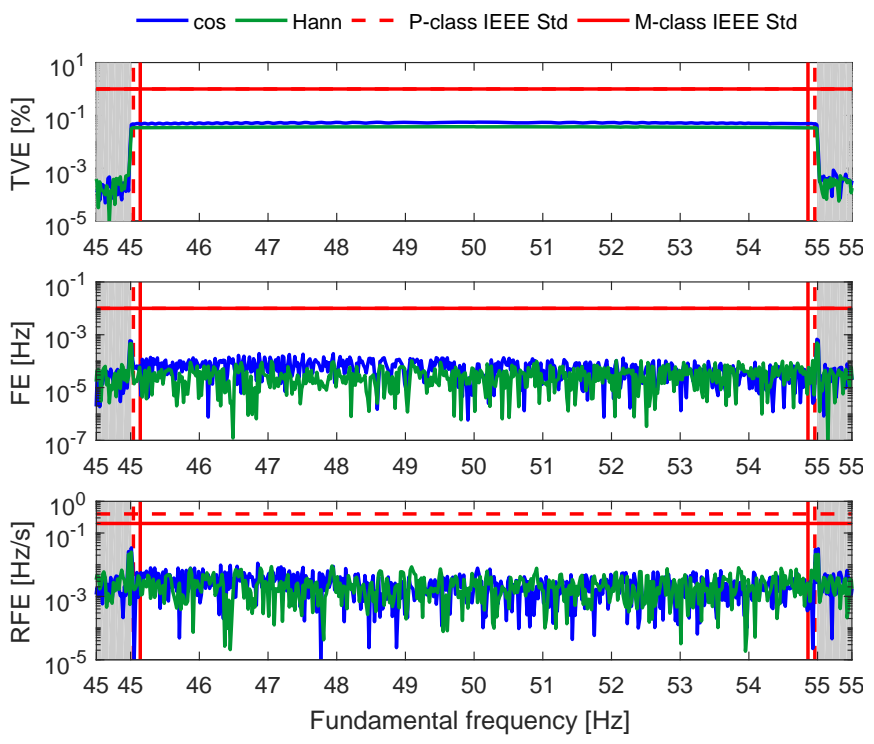

Fig. 13. Frequency ramp test when the signal frequency ramps over the range $[45,55] \mathrm{Hz}$ (positive) [1] (similar results hold for negative frequency ramp and for the $[48,52] \mathrm{Hz}$ range). The gray areas correspond to the initial and final steady-state conditions. The vertical lines delineate the exclusion intervals: $2 / F_{s}=0.04 \mathrm{~s}$ for P-class (dashed lines) and $7 / F_{s}=0.14 \mathrm{~s}$ for M-class (solid lines).
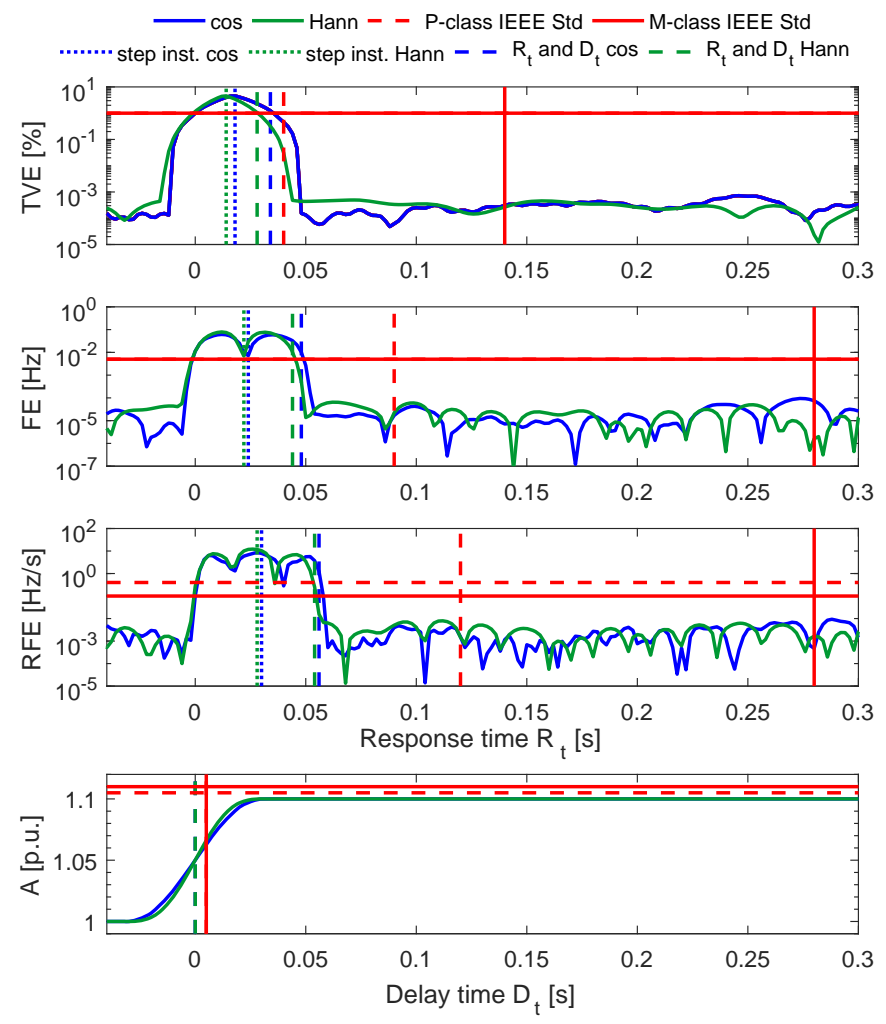

Fig. 14. Amplitude step test (positive) [1] (similar results hold for negative step test). TVE, FE and RFE are represented as a function of the response time, meaning that time $t=0$ corresponds to the instant that the measurement leaves the accuracy limit, to which the curves for the two windows are aligned. The vertical dotted lines indicate the instant that the step change is applied, the vertical dashed lines indicate the SE response time, the vertical red lines indicate the response time limits dictated by [1]. The estimated amplitude is represented as a function of the delay time, meaning that time $t=0$ corresponds to the instant that the step change is applied. The vertical dashed lines indicate the SE delay time, the vertical red lines indicate the delay time limits dictated by [1].
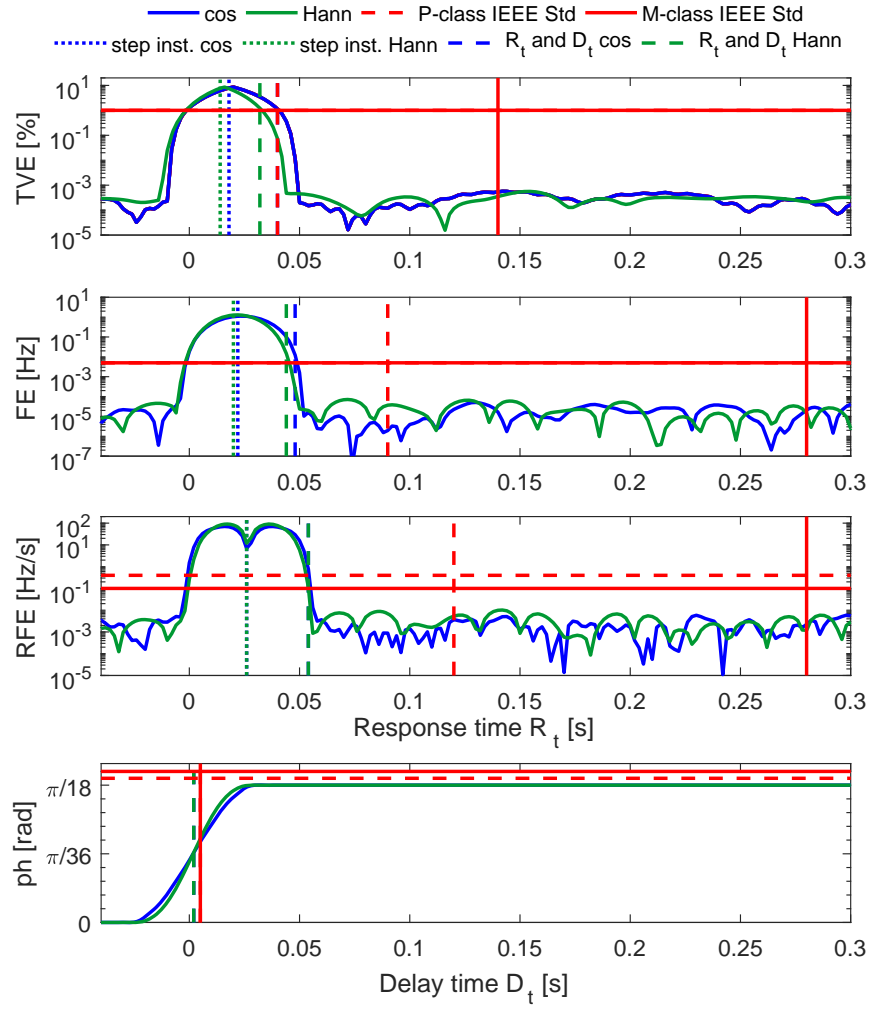

Fig. 15. Phase step test (positive) [1] (similar results hold for negative step test). Refer to Fig. 14 caption for further details.

TABLE IV

COMPUTATIONAL COMPLEXITY OF THE I-IPDFT.

\begin{tabular}{|c|c|c|}
\hline & i-IpDFT Variable & Value \\
\hline & $\begin{array}{l}K \\
P \\
Q\end{array}$ & $\begin{array}{c}11 \\
2 \\
16(\cos ), 28(\text { Hann })\end{array}$ \\
\hline Function & $+|-| x$ & $\div|\exp | \sin$ \\
\hline IpDFT & 14 & 3 \\
\hline $\mathrm{wf}(\cos )$ & $18 K$ & $11 K$ \\
\hline wf (Hann) & $23 K$ & $16 K$ \\
\hline e-IpDFT & $(P+1) \cdot \operatorname{IpDFT}+P \cdot \mathrm{wf}+K P$ & $(P+1) \cdot \operatorname{IpDFT}+P \cdot \mathrm{wf}$ \\
\hline Alg. II & $+|-| \times$ & $\div|\exp | \sin$ \\
\hline line 2 & e-IpDFT & e-IpDFT \\
\hline line 3 & $K+2 \cdot \mathrm{wf}$ & $2 \cdot \mathrm{wf}$ \\
\hline line 4 & $5 K-2$ & - \\
\hline line 6,8 & $Q \cdot \mathrm{e}-\mathrm{IpDFT}+K$ & $Q \cdot \mathrm{e}-\mathrm{IpDFT}$ \\
\hline line 7,9 & $Q \cdot(K+2 \cdot \mathrm{wf})$ & $Q \cdot(2 \cdot \mathrm{wf})$ \\
\hline
\end{tabular}

and the interfering tones. As a consequence, the expected computational latency of the i-IpDFT on the same FPGA target of [22] for a single channel can be approximately computed as a function of the e-IpDFT latency. Specifically, this leads to $20 \mu \mathrm{s} \cdot 2 \cdot 16=0.64 \mathrm{~ms}$ for the cosine and $20 \mu \mathrm{s} \cdot 2 \cdot 28=1.12 \mathrm{~ms}$ for the Hanning window. Those values are fully compatible with the $10 \mathrm{~ms}$ time budget at a reporting rate of $50 \mathrm{fps}$, demonstrating the feasibility of the i-IpDFT technique implementation on an FPGA target similar the one used in [22]. 


\section{CONCLUSIONS}

In this paper, we have presented an IpDFT-based SE algorithm, called i-IpDFT, that is an enhancement of the methods proposed in [7] and [8]. We have discussed the limits of the IpDFT technique when estimating the parameters of a signal corrupted by an interharmonic tone and formulated a technique that is resilient against the spectral leakage generated by any interfering tone. We have conducted a sensitivity analysis to characterize the dependence upon the adopted window function and upon the algorithm's parameters. We have assessed its performance with respect to all the operating conditions introduced for $\mathrm{P}$ and M-class PMUs in [1]. We have presented the results in the case of a power system operating at a nominal frequency of $50 \mathrm{~Hz}$, a reporting frequency of $50 \mathrm{fps}$ and signals characterized by an SNR in the range from 60 to $80 \mathrm{~dB}$.

The proposed i-IpDFT algorithm satisfies all the accuracy requirements defined in [1] for $\mathrm{P}$ and M-class PMUs. In particular, the i-IpDFT fulfils the OOBI test constraints, which is the main noticeable advantage when comparing it with the method presented in [7]. In such an operating condition, the maximum TVE is $0.1 \%$ (being $1.3 \%$ the maximum limit allowed by [1]) and the maximum $\mathrm{FE}$ is $5.6 \mathrm{mHz}$ (being $10 \mathrm{mHz}$ the maximum limit allowed by [1]). Moreover, we have assessed its computational complexity and have demonstrated the prospective deployability of the proposed method in an embedded device.

\section{APPENDIX}

\section{Solution of the IpDFT Problem using the Cosine Window}

The analytical formulation of the IpDFT correction term $\delta$ for the cosine window given in (15) is derived in [20]. Regarding the tone's amplitude given in (16), no derivation was found in the existing literature.

If the number of samples $N$ is sufficiently large, the following approximation is valid [21]:

$$
e^{ \pm j \frac{\pi}{2}(N-1) / N} \approx \pm j
$$

Moreover, sine functions are approximated by their arguments in the case of small angles. The Dirichlet kernel evaluated in $k \pm 0.5$ can be then approximated as:

$$
\begin{aligned}
D_{N}(k \pm 0.5) & =\mp j e^{-j \pi k(N-1) / N} \frac{\sin (\pi k \pm \pi / 2)}{\sin (\pi(k \pm 0.5) / N)} \\
& \approx-\frac{j N}{\pi} e^{-j \pi k(N-1) / N} \frac{\cos (\pi k)}{k \pm 0.5}
\end{aligned}
$$

Therefore, the DFT of the cosine window in (6) can be approximated as:

$$
W_{C}(k)=-\frac{N}{2 \pi} e^{-j \pi k(N-1) / N} \frac{\cos (\pi k)}{k^{2}-0.25}
$$

The tone's amplitude $A_{0}$ corresponds to the modulus of the DTFT of the signal evaluated at frequency $f_{0}=k_{m}+\delta$ :

$$
A_{0}=\left|X\left(k_{m}+\delta\right)\right|
$$

Its value can be derived as a function of the highest amplitude DFT bin:

$$
\begin{aligned}
\frac{\left|X\left(k_{m}+\delta\right)\right|}{\left|X\left(k_{m}\right)\right|} & \approx \frac{\left|W_{C}(0)\right|}{\left|W_{C}(-\delta)\right|} \\
& =4 \cdot \frac{\left|\delta^{2}-0.25\right|}{|\cos (\pi \delta)|}
\end{aligned}
$$

leading to (16). The approximation sign is used instead of equality, because the ratios might differ due to spectral leakage [21].

\section{REFERENCES}

[1] "IEEE Standard for Synchrophasor Measurements for Power Systems," IEEE Std C37.118.1-2011 (Revision of IEEE Std C37.118-2005), pp. 1-61, Dec 2011.

[2] "IEEE Standard for Synchrophasor Measurements for Power Systems - Amendment 1: Modification of Selected Performance Requirements," IEEE Std C37.118.1a-2014 (Amendment to IEEE Std C37.118.1-2011), pp. 1-25, April 2014.

[3] "Interharmonics in power systems," online available: http://grouper ieee.org/groups/harmonic/iharm/, IEEE Interharmonic Task Force, Cigré 36.05/CIRED 2 CC02 Voltage Quality Working Group, Accessed: 201705-01.

[4] A. J. Roscoe, "Exploring the relative performance of frequency-tracking and fixed-filter phasor measurement unit algorithms under C37.118 test procedures, the effects of interharmonics, and initial attempts at merging P-class response with M-class filtering," IEEE Transactions on Instrumentation and Measurement, vol. 62, no. 8, pp. 2140-2153, Aug 2013.

[5] P. Castello, J. Liu, C. Muscas, P. A. Pegoraro, F. Ponci, and A. Monti, "A fast and accurate PMU algorithm for $\mathrm{P}+\mathrm{M}$ class measurement of synchrophasor and frequency," IEEE Transactions on Instrumentation and Measurement, vol. 63, no. 12, pp. 2837-2845, Dec 2014.

[6] M. Pignati, L. Zanni, P. Romano, R. Cherkaoui, and M. Paolone, "Fault detection and faulted line identification in active distribution networks using synchrophasors-based real-time state estimation," IEEE Transactions on Power Delivery, vol. 32, no. 1, pp. 381-392, Feb 2017.

[7] P. Romano and M. Paolone, "Enhanced interpolated-DFT for synchrophasor estimation in FPGAs: Theory, implementation, and validation of a PMU prototype," IEEE Transactions on Instrumentation and Measurement, vol. 63, no. 12, pp. 2824-2836, Dec 2014.

[8] A. Derviškadić, P. Romano, and M. Paolone, "Iterative-interpolated DFT for synchrophasor estimation in M-class compliant PMUs," in 2017 IEEE Manchester PowerTech, June 2017, pp. 1-6.

[9] D. Belega and D. Petri, "Sine-wave parameter estimation by interpolated DFT method based on new cosine windows with high interference rejection capability," Digital Signal Processing, vol. 33, pp. 60 - 70, 2014.

[10] D. Agrez, "Weighted multipoint interpolated DFT to improve amplitude estimation of multifrequency signal," IEEE Transactions on Instrumentation and Measurement, vol. 51, no. 2, pp. 287-292, Apr 2002.

[11] D. Belega, D. Petri, and D. Dallet, "Frequency estimation of a sinusoidal signal via a three-point interpolated DFT method with high image component interference rejection capability," Digital Signal Processing, vol. 24, pp. 162-169, 2014.

[12] D. Belega and D. Petri, "Accuracy analysis of the multicycle synchrophasor estimator provided by the interpolated DFT algorithm," IEEE Transactions on Instrumentation and Measurement, vol. 62, no. 5, pp. 942-953, May 2013.

[13] T. Radil, P. M. Ramos, and A. C. Serra, "New spectrum leakage correction algorithm for frequency estimation of power system signals," IEEE Transactions on Instrumentation and Measurement, vol. 58, no. 5, pp. 1670-1679, 2009.

[14] M. Bertocco, G. Frigo, C. Narduzzi, C. Muscas, and P. A. Pegoraro, "Compressive sensing of a Taylor-Fourier multifrequency model for synchrophasor estimation," IEEE Transactions on Instrumentation and Measurement, vol. 64, no. 12, pp. 3274-3283, Dec 2015.

[15] A. Ferrero, S. Salicone, and S. Toscani, "A fast, simplified frequencydomain interpolation method for the evaluation of the frequency and amplitude of spectral components," IEEE Transactions on Instrumentation and Measurement, vol. 60, no. 5, pp. 1579-1587, May 2011. 
[16] D. Belega, D. Petri, and D. Dallet, "Impact of harmonics on the interpolated DFT frequency estimator," Mechanical Systems and Signal Processing, vol. 6667, pp. 349 - 360, 2016.

[17] V. K. Jain, W. L. Collins, and D. C. Davis, "High-accuracy analog measurements via interpolated FFT," Instrumentation and Measurement, IEEE Transactions on, vol. 28, no. 2, pp. 113-122, 1979.

[18] T. Grandke, "Interpolation algorithms for Discrete Fourier Transforms of weighted signals," Instrumentation and Measurement, IEEE Transactions on, vol. 32, no. 2, pp. 350-355, 1983.

[19] F. J. Harris, "On the use of windows for harmonic analysis with the Discrete Fourier Transform," Proceedings of the IEEE, vol. 66, no. 1, pp. 51-83, 1978.

[20] K. Duda and S. Barczentewicz, "Interpolated DFT for $\sin ^{\alpha}(x)$ windows," IEEE Transactions on Instrumentation and Measurement, vol. 63, no. 4, pp. 754-760, April 2014.

[21] M. Paolone and P. Romano, Advanced Techniques for Power System Modelling, Control and Stability Analysis. F. Milano, IET, 2015, ch. DFT-based synchrophasor estimation processes for Phasor Measurement Units applications: algorithms definition and performance analysis.

[22] P. Romano and M. Paolone, "An enhanced interpolated-modulated sliding DFT for high reporting rate PMUs," in 2014 IEEE International Workshop on Applied Measurements for Power Systems Proceedings (AMPS), Sept 2014, pp. 1-6.

[23] D. Macii, D. Fontanelli, G. Barchi, and D. Petri, "Impact of acquisition wideband noise on synchrophasor measurements: A design perspective," IEEE Transactions on Instrumentation and Measurement, vol. 65, no. 10, pp. 2244-2253, Oct 2016.

[24] "IEEE Guide for Synchronization, Calibration, Testing, and Installation of Phasor Measurement Units (PMUs) for Power System Protection and Control," IEEE Std C37.242-2013, pp. 1-107, March 2013.

[25] G. Barchi, D. Macii, and D. Petri, "Synchrophasor estimators accuracy: A comparative analysis," IEEE Transactions on Instrumentation and Measurement, vol. 62, no. 5, pp. 963-973, May 2013.

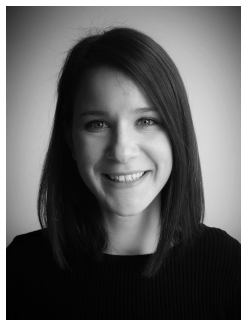

Asja Derviškadić (M'15) was born in Sarajevo, Bosnia and Herzegovina, in 1990. She received the B.Sc. and M.Sc. degrees (with honors) in electrical engineering from the University of Rome "La Sapienza," Rome, Italy, in 2012 and 2015, respectively. She is currently pursuing the Ph.D. degree with the Distributed Electrical System Laboratory (DESL) of the Swiss Federal Institute of Technology of Lausanne (EPFL), Switzerland. Her research interests include the development of enhanced Phasor Measurements Units (PMUs) for active distribution

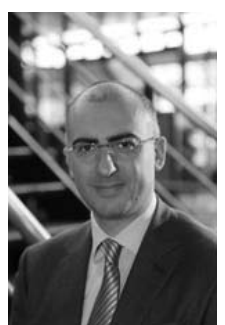

Mario Paolone (M07 - SM10) received the M.Sc. (with honors) and the Ph.D. degree in electrical engineering from the University of Bologna, Italy, in 1998 and 2002, respectively. In 2005, he was appointed assistant professor in power systems at the University of Bologna where he was with the Power Systems laboratory until 2011. In 2010, he received the Associate Professor eligibility from the Politecnico di Milano, Italy. Since 2011 he joined the Swiss Federal Institute of Technology, Lausanne, Switzerland, where he is now Full Professor, Chair of the Distributed Electrical Systems laboratory and Head of the Swiss Competence Center for Energy Research (SCCER) FURIES (Future Swiss Electrical infrastructure). He was co-chairperson of the technical programme committees of the 9th edition of the International Conference of Power Systems Transients (IPST 2009) and of the 2016 Power Systems Computation Conference (PSCC 2016). He is chair of the technical programme committee of the 2018 Power Systems Computation Conference (PSCC 2018). In 2013, he was the recipient of the IEEE EMC Society Technical Achievement Award. He was co-author of several papers that received the following awards: best IEEE Transactions on EMC paper award for the year 2017, in 2014 best paper award at the 13th International Conference on Probabilistic Methods Applied to Power Systems, Durham, UK, in 2013 Basil Papadias best paper award at the 2013 IEEE PowerTech, Grenoble, France, in 2008 best paper award at the International Universities Power Engineering Conference (UPEC). $\mathrm{He}$ is the Editor-in-Chief of the Elsevier journal Sustainable Energy, Grids and Networks and Associate Editor of the IEEE Transactions on Industrial Informatics. His research interests are in power systems with particular reference to real-time monitoring and operation, power system protections, power systems dynamics and power system transients. Mario Paolone is author or coauthor of over 220 scientific papers published in reviewed journals and international conferences.

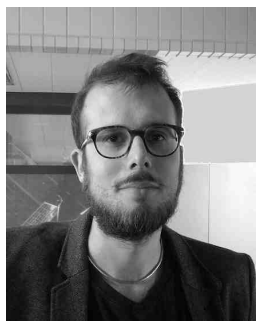

Paolo Romano (M'12) is a Postdoctoral fellow at the Distributed Electrical Systems Laboratory (DESL) of the Swiss Federal Institute of Technology of Lausanne (EPFL). He received his B.Sc. and M.Sc. degree (with honors) in electronics engineering from the University of Genova, Italy, in 2008 and 2011 respectively, and his Ph.D. from the Swiss Federal Institute of Technology of Lausanne in 2016. His research interests refer to the synchrophasor area and particularly to the development of advanced Phasor Measurement Units for the real-time monitoring of active distribution networks. 